\title{
Simulation and Analysis of Si Schottky Diode Family in DC-DC Converter
}

\author{
N. Z. Yahaya ${ }^{1}$, F. H. Ramle ${ }^{2}$ \\ Electrical Engineering Department, Universiti Teknologi PETRONAS \\ Bandar Seri Iskandar, Tronoh 31750 Perak, MALAYSIA \\ 1norzaihar_yahaya@petronas.com.my \\ ${ }^{2}$ fairuz_hanisah@utp.edu.my
}

\begin{abstract}
The unipolar-based devices, silicon schottky ( $\mathrm{Si}$ ) and silicon carbide schottky (SiC) power diodes are investigated for their reverse recovery transient responses and the effects on the switching losses of the FET in the DC-DC converter. Two inductive load chopper circuits are simulated with different test diodes using PSpice software to determine the effectiveness and superiority in each of the devices. All parameter settings are consistent for both converter circuits. The results have shown that the $\mathrm{SiC}$ diode has higher energy savings of more than $73.13 \%$ in turn-off switching losses. This eventually corresponds to the reduction of $96.16 \%$ in FET turn-on peak power dissipation of $\mathrm{SiC}$ converter. However, there is a minor drawback in drain voltage overshoot of the converter. Some detailed analyses are presented in the paper.
\end{abstract}

Keywords: Power Diode, PSpice, Reverse Recovery, Silicon Carbide Schottky, Silicon Schottky

\section{Introduction}

Semiconductor power devices, especially diodes play important role in switching transient response. Low power dissipation on the switching device will give rise to highly efficient power electronic system. For example, if a semiconductor device operates in a linear mode such as in power amplifiers or in linear regulators, it is expected that some amount of energy will be lost in the power circuit before its energy reaches the output. High efficiency of power converter requires a minimal loss of this energy driven from source to load. One of the energy can be easily dissipated in the diodes in terms of heat leading to a lower efficiency of the converter [1].

The unipolar Silicon schottky ( $\mathrm{Si}$ ) and Silicon carbide schottky ( $\mathrm{SiC}$ ) diodes are commonly used in power converter circuits. In spite of both diodes come from the same unipolar family, the issues of higher switching losses with regards to reverse recovery losses have yet been solved. Nevertheless, the new $\mathrm{SiC}$ diode has emerged in the market in recent years where they are expected to improve the efficiency of the converter by allowing a further reduction in reverse recovery energy losses and hence increasing the performance. The additional substance of carbide element in the power schottky diode may eventually lower the reverse charge current and thus, improve the overall transient response in the converter.

An ideal semiconductor device would inhibit large breakdown voltage, low voltage drop in the on-state, high switching speed and low power loss. However, in order to increase the performance of a semiconductor device, several additional doping enhancements will be added during the fabrication stage, where the characteristic of the device will be altered by adding some impurity atoms to the pure semiconductor material. Today's technology requires extensive research to develop more powerful devices, not only to have lower switching losses, higher efficiency but also improve reliability. For this reason, power losses in the device must be put into consideration. The main contribution in this work is to verify that $\mathrm{SiC}$ high power diode having larger energy bandgap can indeed produce better results in DC-DC converter. The analyses are mainly done using circuit simulator.

Received: October 5, 2009. Accepted: November 30, 2009 


\section{Silicon Schottky Diode Family}

The Silicon schottky diode or Schottky barrier diode is widely used as a mixer or detector diode. In addition, due to its low forward voltage drop, it may lead to lower levels of power losses in the circuit [2]. Since Si diode is a unipolar device, the current transport is mainly due to majority carriers and therefore the speed is faster. In addition, it has low turn-on voltage, high frequency capability and low capacitive effect [3]. SiC diode is a wide-bandgap (WBG) semiconductor family from III-V group. It has the advantage in a faster recovery times as well as no dependence on temperature [4] and has the potential to operate more efficiently. In Si diode however, the temperature may rise due to the increasing in electron's thermal energy leading to higher peak reverse recovery current [5]. This eventually gives higher power loss in the device.

In addition to smaller package and lighter weight, $\mathrm{SiC}$ diode also has higher critical field and barrier heights compared to Si diode. This results in reduced on-state resistance and lower leakage current of $\mathrm{SiC}$ diode [6]. It also has been demonstrated that the $\mathrm{SiC}$ diode has the potential to improve power FET performance [7]. Its energy bandgap is three times higher than $\mathrm{Si}$ and ten times higher in electrical breakdown strength. Therefore, $\mathrm{SiC}$ can operate at operating voltage of 20 times with current densities up to 400 times higher than Si diode [8].

Si schottky diode has a small forward voltage and the reverse breakdown voltage cannot be made too high (currently about $200 \mathrm{~V}$ ). It is used for rectification of power supplies for low voltage and high current applications or in high frequency systems due to its small reverse recovery time.

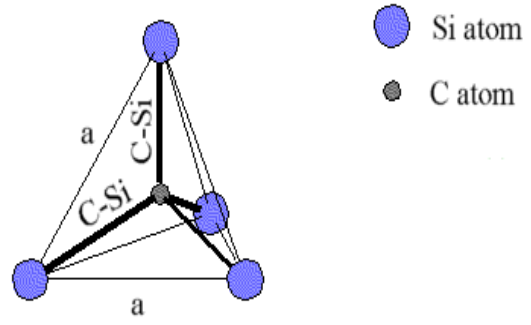

Fig. 1 Tetra Bonding of a Carbon Atom with Four nearest Silicon Neighbours [9]

Fig. 1 shows four $\mathrm{Si}$ atoms made a covalent bonding with a single Carbon (C) atom in order to form a $\mathrm{SiC}$. The $\mathrm{C}$ atom is located in the middle of the structure and the distances between all atoms which marked $\mathrm{C}-\mathrm{Si}$ are equal. The $\mathrm{SiC}$ possesses increase tolerance to radiation damage, making it a material suitable for defence and aerospace applications. Due to high tolerance of temperature in $\mathrm{SiC}$, it is chosen in various industries, such as aircraft, automotive, communications, power and spacecraft.

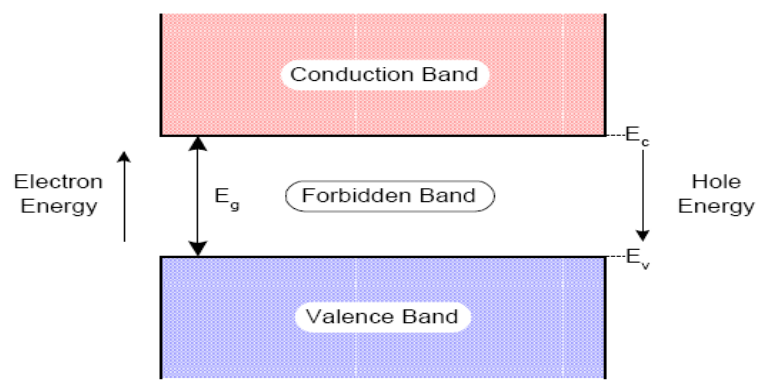

Fig. 2 Energy Band Diagram of a Semiconductor [10] 
The characteristic of $\mathrm{SiC}$ diode as a wide bandgap semiconductor device results in a more energy to excite the electron from its covalent bond during turn-off compared to Si. Referring to Fig. 2, the wide bandgap is measured from the distance between the conduction band and valence band. An insulator would have a larger bandgap that would take lots of energy for the electrons to travel from the valence to conduction band while a conductor would have no forbidden band. The energy is calculated from the difference between both bands. The wider the bandgap, more thermal energy is required to excite the electrons enabling the device to operate at higher temperature without affecting its electrical properties.

\section{Reverse Recovery}

Reverse recovery is one of the properties in a diode. It can be a factor in determining the efficiency of the applications. When a diode has been conducting in a forward bias long enough for it to establish steady state, there will be charges due to the presence of minority charge carriers. This charge must be removed to block in reverse direction [11].

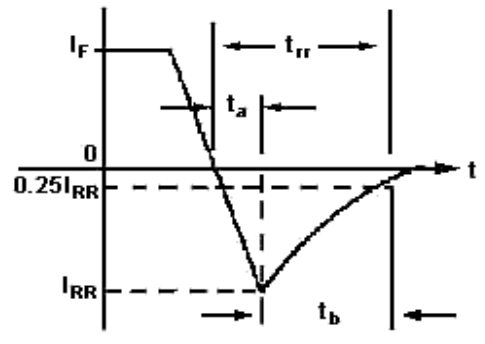

(a) Soft recowery

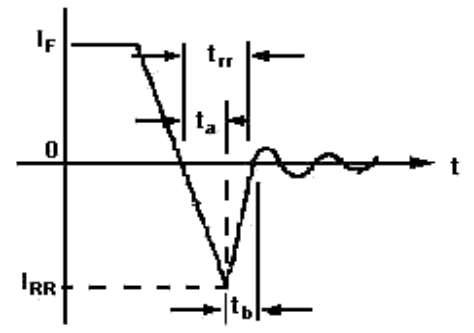

(b) Abrupt recowery

(b) Abrupt recovery

(a) Soft recovery

Characteristic [11]

The characteristic of reverse recovery current experienced by a diode is represented in Fig. 3 above. $t_{r r}$ represents the reverse recovery time, $I_{r r}$ is the peak reverse current whilst $t_{a}$ is the transition time due to charge stored in depletion region of the $\mathrm{p}-\mathrm{n}$ junction. $t_{b}$ is the time for the current to relax to zero. The peak reverse recovery current depends on the falling rate of change in current during turn-off. In $\mathrm{SiC}$ diode, there will be less or none reverse recovery current due to its ability to immediately remove stored charge. However, there are differences observed during the recovery from the peak values. This is merely reflected from different device's fabrication techniques.

Normally, in $\mathrm{SiC}$, the rising current rate to zero takes a longer time $\left(t_{r r}\right)$ as shown in part (a) in Fig. 3. This eventually reduces the turn-off speed. In other SiC type, the speed can also be slightly faster due to smaller $t_{b}$ but with the cost of higher dissipation. This can be seen in part (b) as oscillation exists during the end stage of turn-off time. In addition, if the falling current rate during the beginning of turn-off time is high as in the case of non-schottky diode, the reverse current would also be high, leading to both high power dissipation and lower in turn-off speed. 


\section{Diode Characteristics}

\section{A. Static Characteristic}

The I-V and reverse current are among the static characteristics of the device. Due to higher level of majority carrier injection in Si diode, this causes a lower voltage drop and hence smaller capacitance to bias the junction for turn-on process. This is the only advantage of $\mathrm{Si}$ diode compared to $\mathrm{SiC}$. Here, $\mathrm{SiC}$ diode requires a higher voltage to forward bias the device [12]. Apart from that, $\mathrm{SiC}$ diode can handle larger reverse voltage as compared to $\mathrm{Si}$.

\section{B. Dynamic Characteristic}

The characteristic that changes with time is inherited in both devices. Si and $\mathrm{SiC}$ diodes are compared in terms of the reverse recovery time, reverse recovery current and corresponding switching losses. The comparisons in dynamic characteristics between two devices are tabulated in Table I [13]. The SiC and Si diodes used are of part number SDP04S60 and SB30$03 \mathrm{~F}$ respectively.

Table I

Comparison of Dynamic Characteristics

\begin{tabular}{|l|l|l|}
\hline \multicolumn{1}{|c|}{ Characteristics } & \multicolumn{1}{|c|}{$\begin{array}{c}\text { SiC Schottky } \\
\text { (SDP 04S60) }\end{array}$} & \multicolumn{1}{c|}{$\begin{array}{c}\text { Si Schottky } \\
\text { (SB30-03F) }\end{array}$} \\
\hline Reverse Recovery Time & Unchanged with temperature variation & Increases as temperature increases \\
\hline Reverse Recovery Current & Negligible & Increases as temperature increases \\
\hline Switching Losses & Low & Slightly higher \\
\hline
\end{tabular}

Table I shows that $\mathrm{SiC}$ diode has advantages in all dynamic characteristics. Si diode suffers from higher reverse recovery current and switching losses. This clearly indicates the additional carbide substance in the device may improve switching speed and reduce power dissipation.

\section{Methodology}

Two separate inductive chopper circuits are constructed and simulated in PSpice where each of the diodes under test (DUT) is applied to each of the circuit. The test circuit is shown in Fig. 4. The diodes under test are $D_{1 \_S i C}$ and $D_{2 \_S i}$ for $\mathrm{SiC}$ and Si diodes respectively. Table II shows the list of components used in the test circuit for $\mathrm{SiC}$ diode. The parameters used for the other test circuit for Si diode are the same except for the DUT. There is a resistor in the gate link of the switch. The purpose for this is to drive a lower current to the FET as well as avoid high overshoot which may damage the switch. The switching frequency is applied at $40 \mathrm{kHz}$ with $50 \%$ duty ratio. 
Table II

Device Parameters in Test Circuit

\begin{tabular}{|l|l|l|}
\hline Parameter & Part number & Ratings / Value \\
\hline$M_{1}-\mathrm{N}-$ Ch. FET & IRF520 & $9.2 \mathrm{~A} / 100 \mathrm{~V}$ \\
\hline$D_{1 \_} S i C$ & SDP06S60 & $6 \mathrm{~A} / 600 \mathrm{~V}$ \\
$D_{2 \_} S i$ & SB30-3F & $3 \mathrm{~A} / 30 \mathrm{~V}$ \\
\hline$V_{c c} /$ Temperature & - & $25 \mathrm{~V} / 27^{\circ} \mathrm{C}$ \\
\hline$R_{g 1}$ & - & $21 \Omega$ \\
\hline$R_{\text {load }}$ & - & $55 \Omega$ \\
\hline$L_{\text {load }}$ & - & $500 \mathrm{uH}$ \\
\hline
\end{tabular}

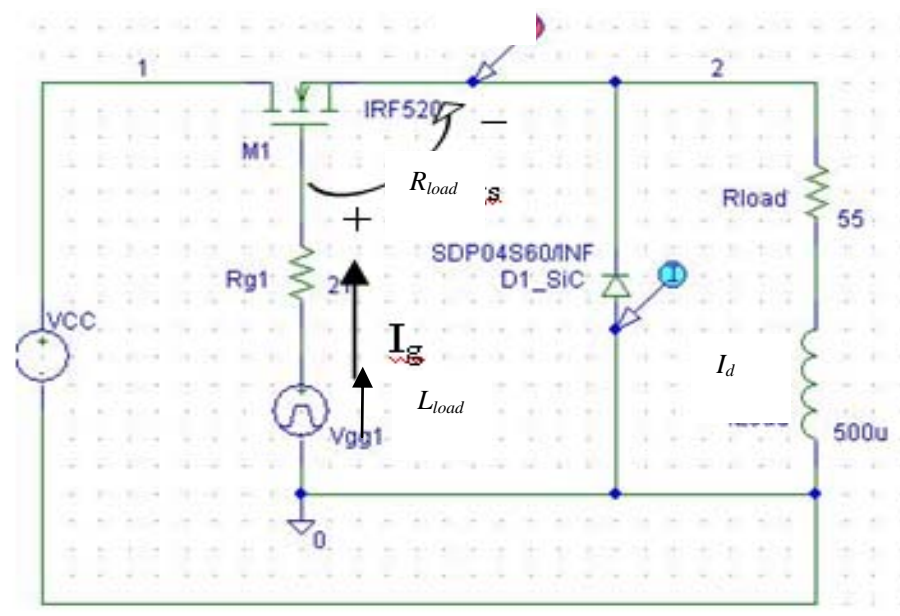

Fig. 4 Inductive Load Chopper Circuit

$V_{g g 1}$ provides a pulse signal to the FET $\left(M_{1}\right)$ and the signal will appear at $V_{g s}$. The pulse signal will then forward bias the gate-source junction of the FET. As a result, the FET is turned on. At this time, current will develop in $L_{\text {load }}$ and increase to its peak value. There is no current flowing in the DUT $\left(D_{1 \_ \text {SiC }}\right)$ yet. When FET is turned off, the charged inductor current will be discharged through the low impedance path, $D_{1_{-} S i C} R l_{\text {oad }}$ and $L_{\text {Load }}$. This process repeats in the next subsequent switching cycle.

Fig. 5 shows the signal waveform of the $\mathrm{V}_{\text {pulse. }}$ It is of the same signal applied for both chopper circuits, having a square wave with pulse period of $25 \mu$ s and $20-\mathrm{V}$ peak voltage. During turn-off of the FET, current will conduct in the diode, leading to forward bias of the device. Once the switch is turned on again, the diode current, $I_{d}$ will be forced to discharge rapidly leading to high voltage overshoot in $M_{1}$ before it goes back to forward voltage value.

In addition, $I_{d}$ will not decay directly to zero forward current value but to a negative peak instead. This negative or reverse overshoot is called reverse recovery of diode current. The longer it takes to recover back to zero will indicate a higher switching loss in the device. Due to this, the switching FET will also experience forward overshoot during its turn-on cycle. In this work, the level of switching losses is evaluated to verify the advantages of $\mathrm{SiC}$ over $\mathrm{Si}$ diode. 
N. Z. Yahaya, et al.

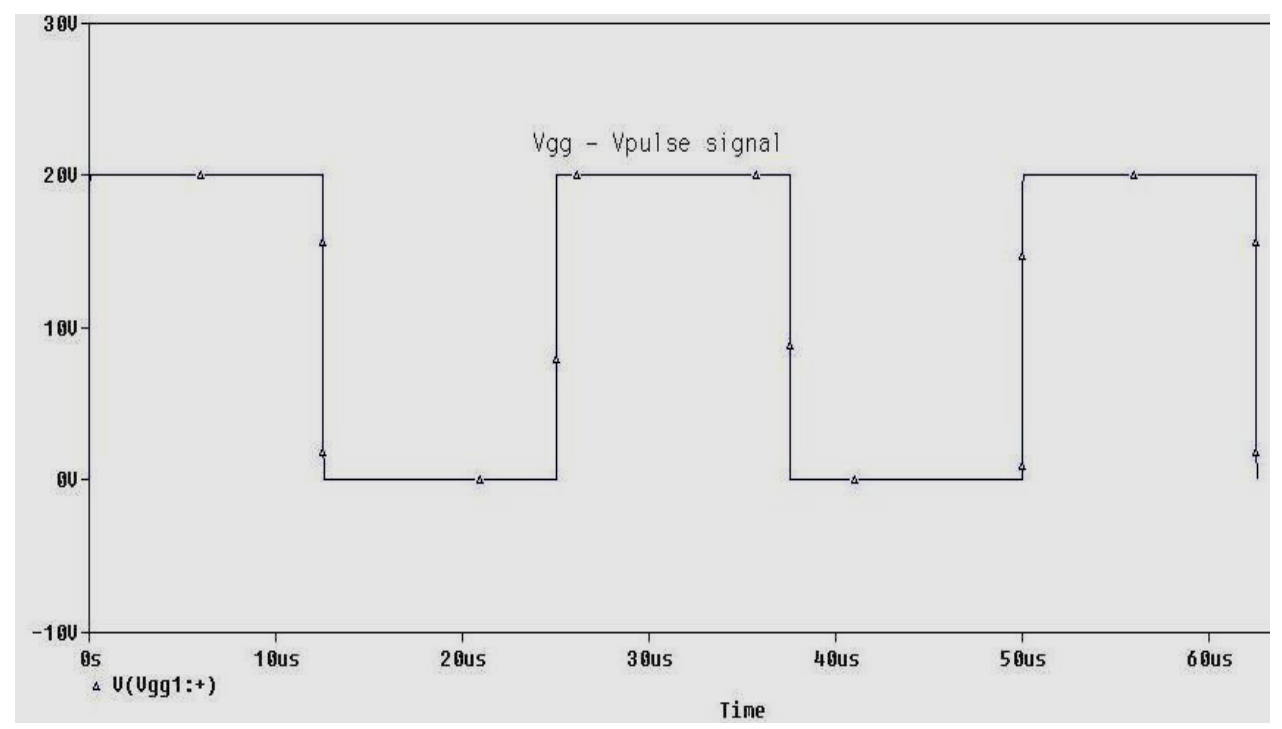

Fig. $5 V_{g g 1}($ Vpulse) Signal

\section{Results and Discussions}

Correct choice of diode is important in understanding its effects on the overall performance in DC-DC converter. One of the effects is the voltage overshoot in the switch. Gate and drain voltages are prone to stress when diode suffers from high reverse recovery and hence may give rise to less switching speed and high dissipation in the circuit. Fig. 6 shows the forward overshoot in $V_{g s}$ of $M_{1}$ for $\mathrm{SiC}$ and $M_{2}$ for $\mathrm{Si}$. It is clearly shown that $\mathrm{Si}$ diode imposes a peak overshoot more than $16 \%(6.02 \mathrm{~V})$ compared to $\mathrm{SiC}$ diode $(5.05 \mathrm{~V})$ in the switch. Here, more heat is dissipated in $\mathrm{Si}$, resulting in higher stress to the switch.

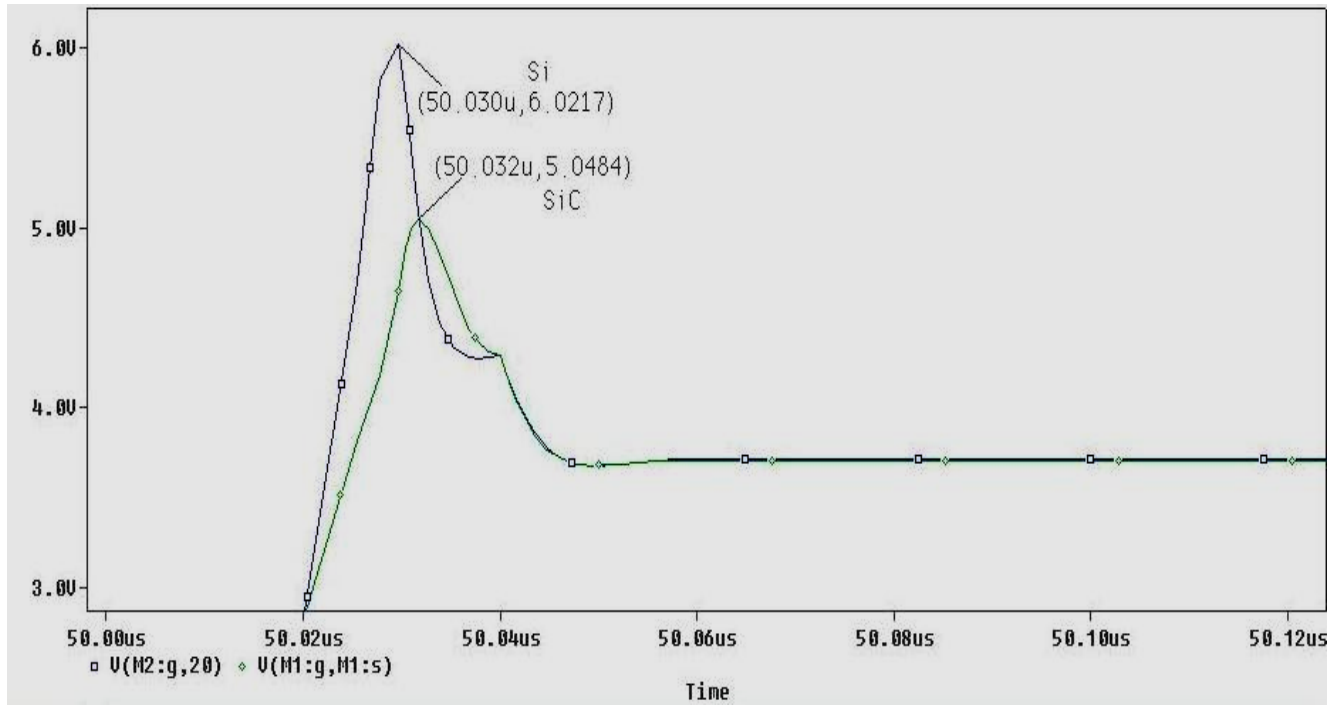

Fig. $6 V_{g s}$ Forward Overshoot of Switch $M_{1}$ for $\mathrm{SiC}$ and $M_{2}$ for Si Diode. 
In addition, as seen in Fig. 7, the FET's $V_{d s}$ overshoot is higher in $\mathrm{SiC}$ diode. This contradictory result makes the only drawback in the device. SiC has an overshoot voltage of $26.96 \mathrm{~V}$, which is $6.23 \%$ higher than Si that is only $25.28 \mathrm{~V}$. This is caused by the charge removal response time in $\mathrm{SiC}$ where $t_{b}$ is higher as previously shown in Fig. 3(a). Nevertheless, this result may not affect the improvement in the circuit because the variation in load circuit design may also cause to this drawback.

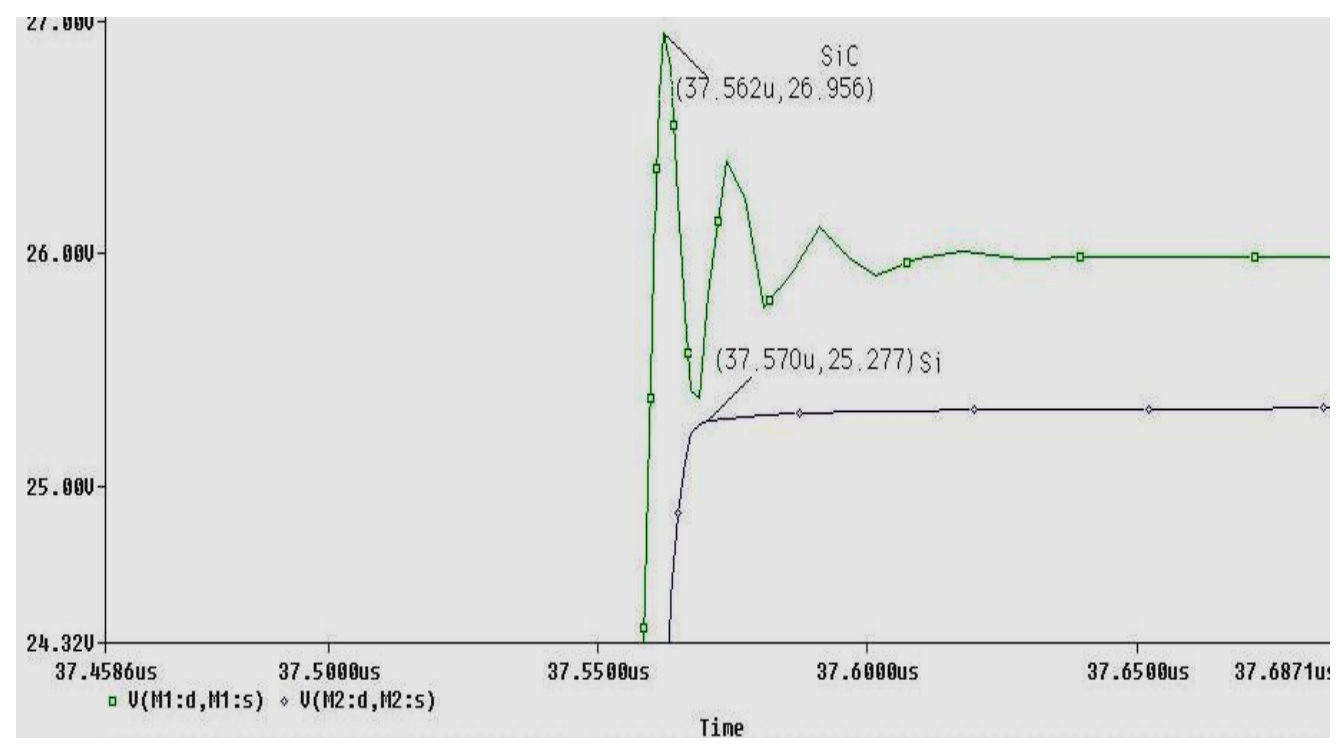

Fig. $7 V_{d s}$ Overshoot of Switch $M_{1}$ for SiC and $M_{2}$ for Si Diode

From gate voltage point of view, Fig. 6 indicates that the switching power loss during turnon of the switch is smaller when $\mathrm{SiC}$ diode is used. The lower the peak overshoot gate voltage gives a clear indication of a lower switching loss and stress in the switch. Fig. 8 shows the load currents of both diodes in the chopper circuit. The maximum swing of the currents is almost identical in value. However, the minimum peak is slightly greater for the $\mathrm{SiC}$ diode by merely $0.91 \%$. From this result, eventually, the output power can be calculated and compared.

In order to calculate the output power, the information such as the minimum and maximum peak current have to be averaged before their squared values can be multiplied with the load resistance. The details are tabulated in Table III.

Even though the FET peak voltage overshoot in $\mathrm{SiC}$ chopper circuit is higher, the corresponding output power, $P_{\text {out }}$ of the circuit is slightly lower. This is due to the smaller peakto-peak swing at the load current. However, the results are acceptable since the difference is just $7.70 \%$. This is another drawback where design compromise has to be made between the output power and reverse recovery current in the diode.

When FET is turned off, load current will start to discharge through the diode and this overshoots the current further in the negative region before it goes back to zero, mainly due to the removal of large amount of charges in the diode. The faster the removal of charge or the smaller of charge appeared in the diode will make the transient response faster. The reverse recovery current of both diodes during FET turn-on is shown in Fig. 9. 
N. Z. Yahaya, et al.

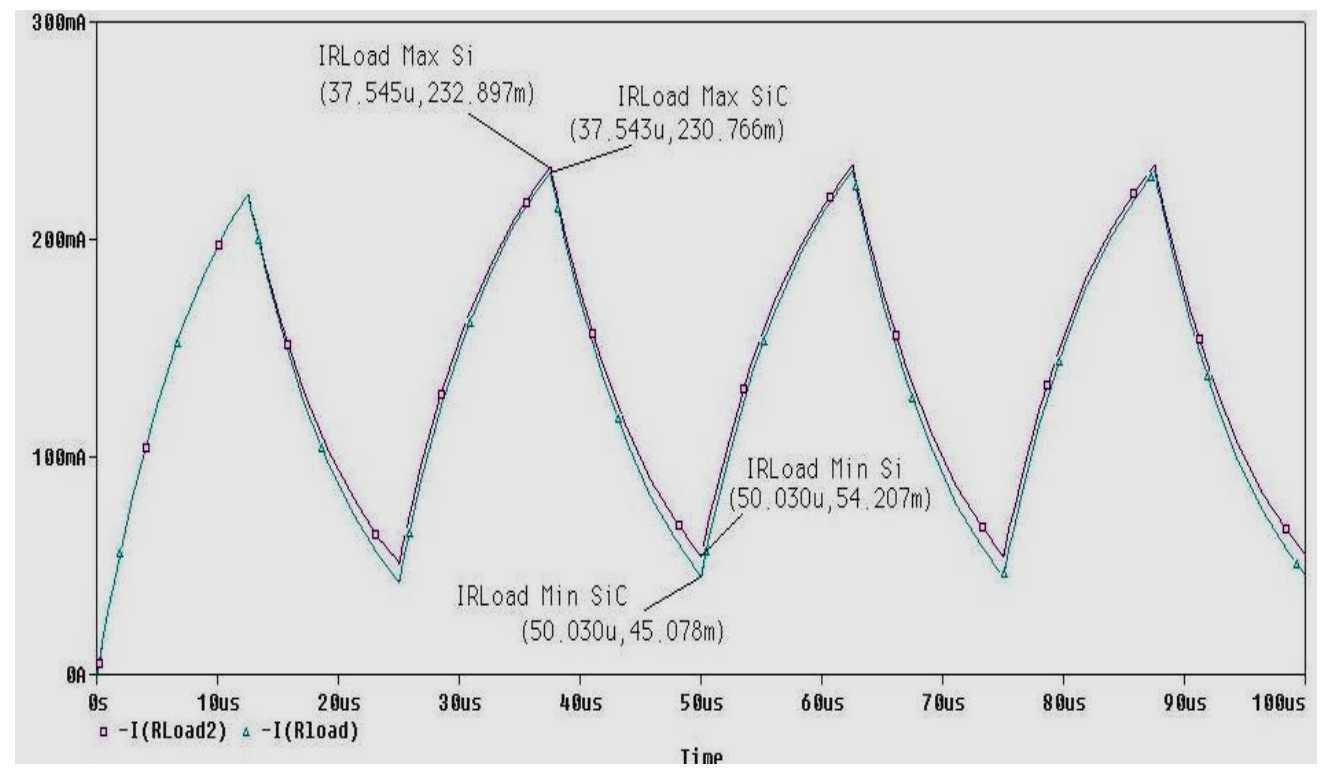

Fig. $8 I_{\text {Rload }}$ for SiC and Si Circuit.

Table III

Comparison in Current and Output Power

\begin{tabular}{|l|l|l|}
\hline$R_{\text {Load }}=55 \Omega$ & Si Schottky & SiC Schottky \\
\hline$I_{\text {RLoad,min }}$ & $54.21 \mathrm{~mA}$ & $45.08 \mathrm{~mA}$ \\
\hline$I_{R \text { Load,max }}$ & $232.90 \mathrm{~mA}$ & $230.77 \mathrm{~mA}$ \\
\hline$I_{R \text { Load,avg }}$ & $143.55 \mathrm{~mA}$ & $137.92 \mathrm{~mA}$ \\
\hline$P_{\text {out }}$ & $1.13 \mathrm{~W}$ & $1.05 \mathrm{~W}$ \\
\hline
\end{tabular}

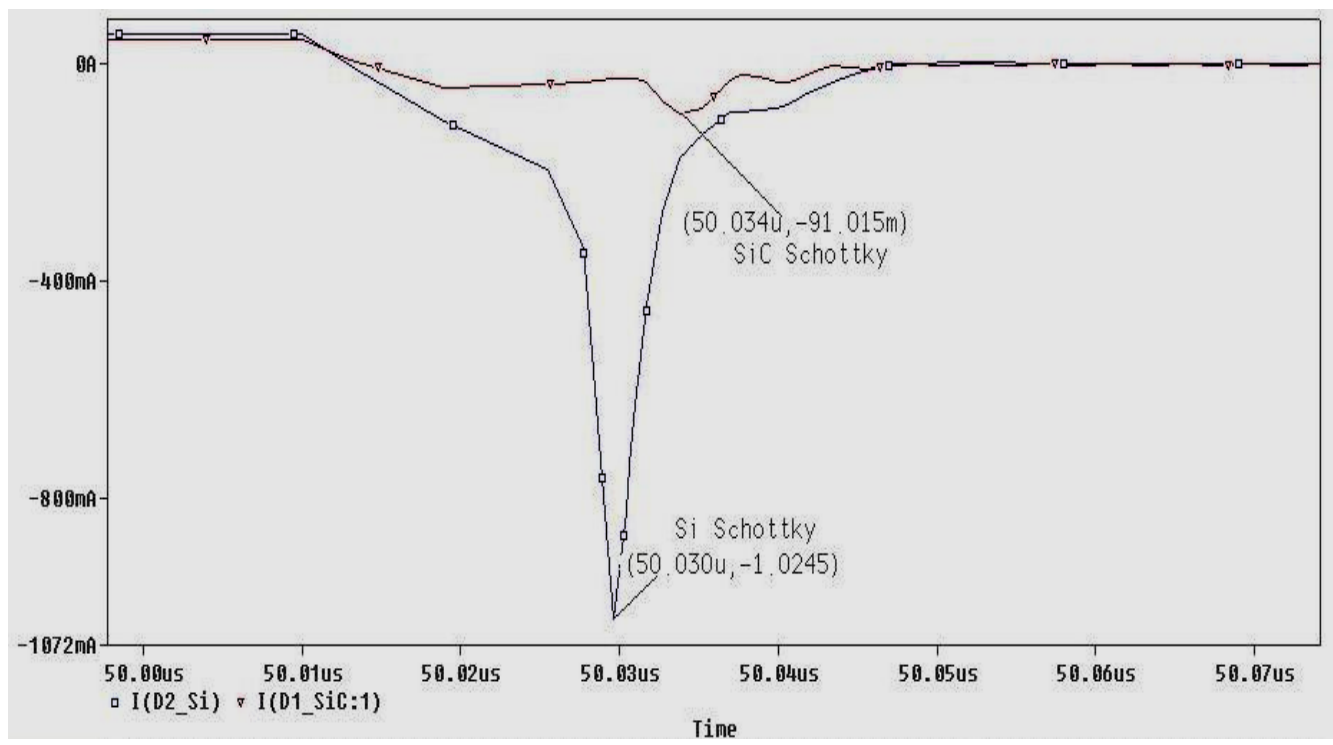

Fig. 9: Reverse Recovery Current of Si and SiC Diodes during FET Turn-on 
From Fig. 9, a significant difference of $91.12 \%$ in reverse recovery current between $\mathrm{SiC}$ diode and Si diode can be observed. There is more charge required to be removed during diode's turn-off for $\mathrm{Si}$, showing higher switching losses [14]. Looking at the $\mathrm{SiC}$ diode, the reverse recovery current is small, indicating an operation without high-level minority carrier injection required, and hence no stored charge to be removed. The low switching losses of $\mathrm{SiC}$ diode is due to the factor of high breakdown field which results in reduced blocking layer thickness, in conjunction to the reduced charges [15]. Due to the facts that SiC structure of metal-semiconductor barrier is two times higher than $\mathrm{Si}$ and has smaller intrinsic carrier concentration, this leads to a lower leakage current from anode to cathode in $\mathrm{SiC}[16,17]$. All of these reasons make $\mathrm{SiC}$ diode a better choice in converter operation.

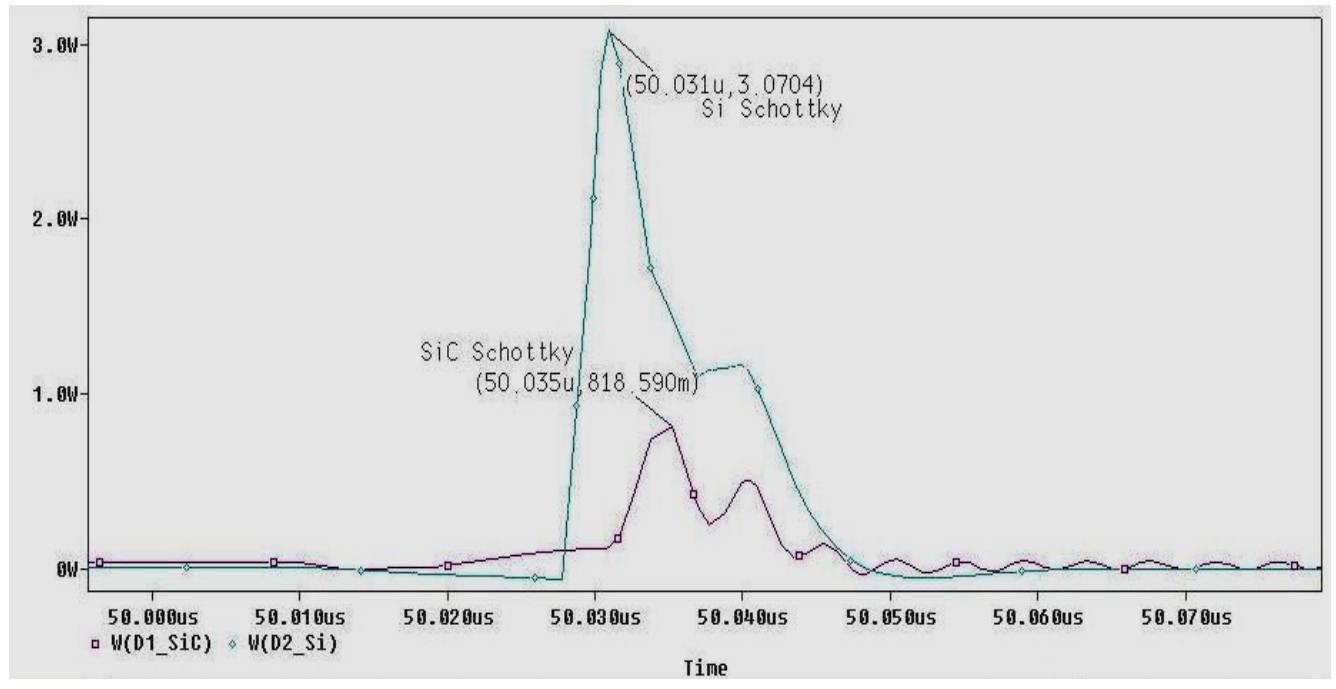

Fig. 10: Turn-Off Loss of Si and SiC Diodes

Fig. 10 shows the turn-off switching losses of $\mathrm{Si}$ and $\mathrm{SiC}$ diodes during FET turn-on. Si diode has a larger peak turn-off loss of more than $73 \%(3.07 \mathrm{~W})$ compared to $\mathrm{SiC}$ diode $(818.59 \mathrm{~mW})$. The result is justified due to higher reverse recovery current seen in Si diode as shown in Fig. 9. With higher reverse recovery current, more power is required to remove the stored charges and hence more losses will be generated before the diode can be fully turned off.

In the inductive chopper circuit, when diode is in off state, FET switch is conducting. Due to the reverse recovery current experienced by the diode, this directly influences the switching losses in the FET switch. In Fig. 11, the peak power loss in FET during turn-on is higher when Si diode is used in the test circuit. This clearly indicates the switching stress in the switch leading to higher power dissipation. There is more than $96 \%$ of stress level in the switch when $\mathrm{Si}$ is used.

It is important to evaluate the significance of $\mathrm{SiC}$ diode of being the right choice for DCDC converter operations. From the simulation work, the diode will go into the reverse recovery stage when the FET switch is turned on. This is when diode undergoes reverse current before it reaches off state. During these conditions, both switch and diode will generate switching losses. However, the output power is slightly less when $\mathrm{SiC}$ is used. 
During FET turn-on, it can be seen that the gate voltage overshoots with some spikes. However, the overshoot level is less when $\mathrm{SiC}$ diode is applied in the test circuit. A lower voltage ringing effect will eventually give lower power loss produced by the FET during turnon. Here, the carbide substance in SiC diode has indeed helped in reducing the FET's stress as well as improving switching loss in the diode.

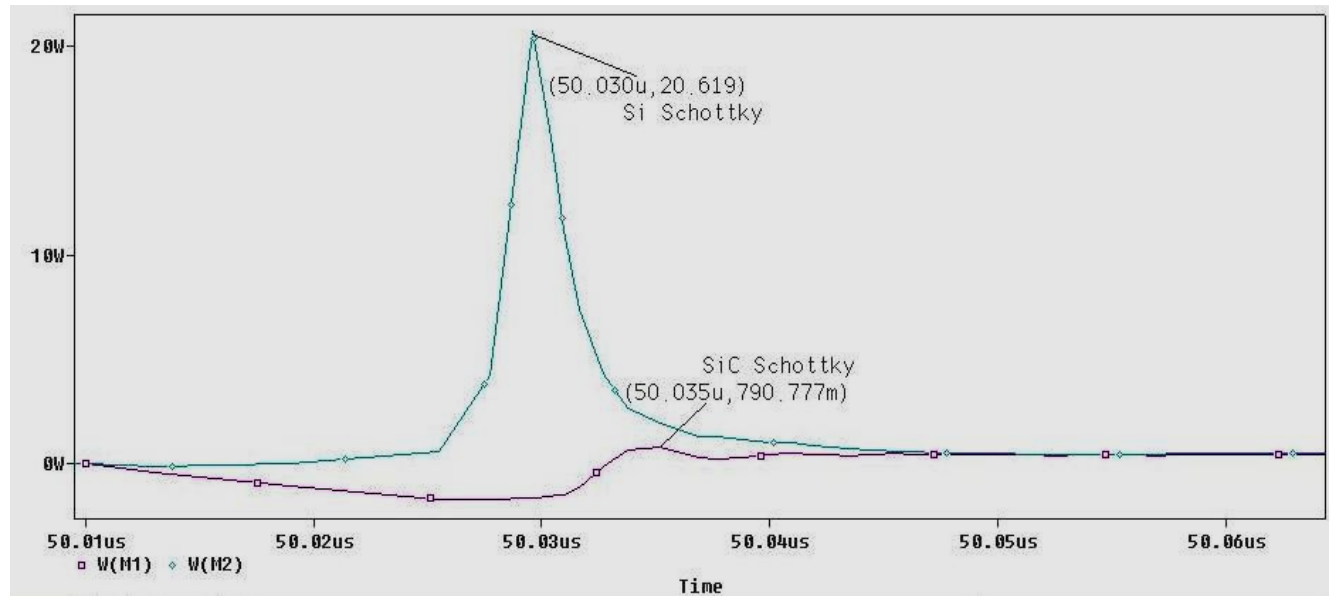

Fig. 11: FET Turn-On Power Loss during DUT Turn-Off

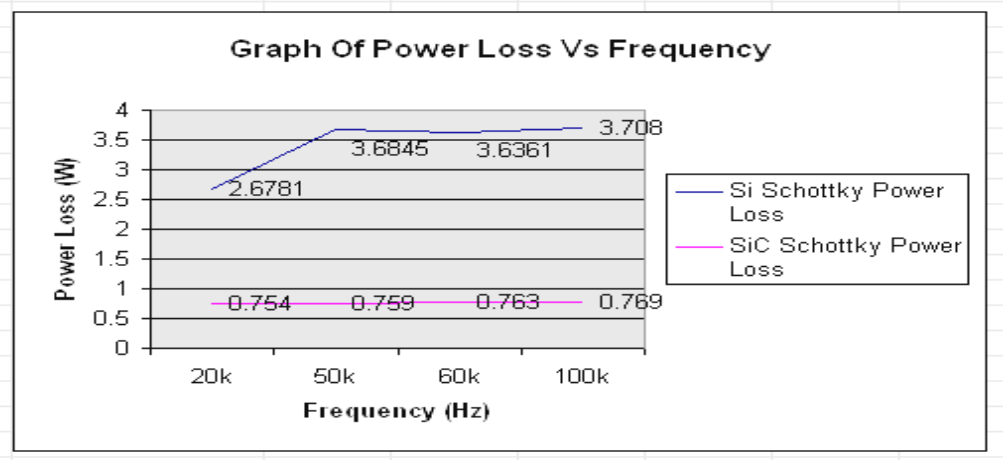

Fig. 12 Power Loss vs Frequency of SiC and Si Diodes

In Fig. 12, when frequency is increased from $20 \mathrm{kHz}$ to $100 \mathrm{kHz}$, the power loss in $\mathrm{Si}$ increases but not to $\mathrm{SiC}$. $\mathrm{SiC}$ does not have a significant difference in variation of frequencies. The increase of power loss in Si relates to the higher reverse recovery current and high falling rate, di/dt during its turn-off. The results have been verified and tabulated in Table IV. 
Table IV

Comparison in Current and Power Losses

\begin{tabular}{|l|l|l|l|}
\hline \multicolumn{1}{|c|}{ Characteristics } & Si Schottky Diode & SiC Schottky Diode & \multicolumn{1}{c|}{$\begin{array}{c}\text { Percentage } \\
\text { Improvement (\%) }\end{array}$} \\
\hline Output Power, $P_{\text {out }}$ & $1.13 \mathrm{~W}$ & $1.05 \mathrm{~W}$ & $-7.69 \%$ \\
\hline $\begin{array}{l}\text { Peak Reverse } \\
\text { Recovery Current, } I_{r r}\end{array}$ & $-1.02 \mathrm{~A}$ & $-91.02 \mathrm{~mA}$ & $91.12 \%$ \\
\hline DUT Turn-Off Loss (peak) & $3.07 \mathrm{~W}$ & $818.59 \mathrm{~mW}$ & $73.33 \%$ \\
\hline FET Peak Turn-On Loss & $20.62 \mathrm{~W}$ & $790.78 \mathrm{~mW}$ & $96.16 \%$ \\
\hline
\end{tabular}

Except for the output power, all of the tested parameters have shown that $\mathrm{SiC}$ diode can give better performance in the circuit. In spite of lower output power and hence lower efficiency, this value is small in difference of only $7.69 \%$. The most significant impacts are less switching losses in the circuit and reduced stress experienced by the FET switch during turn-on. Therefore, the work has shown its major contribution in justifying that $\mathrm{SiC}$ diode is better in the peak reverse recovery current $(91.12 \%)$ and turn-off loss $(73.33 \%)$. Consequently, the FET turn-on stress has been reduced by $96.16 \%$.

\section{Conclusions}

An inductive load chopper circuit is used in the simulation and the output in terms of reverse recovery current turn-off losses of Silicon Carbide Schottky (SiC) and Silicon Schottky (Si) diodes, their forward turn-on losses and losses in the FET during turn-on are analysed and studied. From the simulation, it has shown that Si diode produces higher reverse recovery current than its counterpart, $\mathrm{SiC}$ diode implying more stored charge to be removed. Therefore, higher power losses are dissipated in Si diode by a difference of $91.12 \%$. Even though the output power drops slightly by a margin of $7.69 \%$, the peak power losses in $\mathrm{SiC}$ and switch have been eventually reduced by $73.33 \%$ and $96.16 \%$ respectively in spite of a slight increase in overshoot $V_{d s}$ value of $6.23 \%$. Due to the stronger covalent bonding of carbide substance in $\mathrm{SiC}$ diode, this gives the device a preferred choice for high frequency and high power applications.

\section{Acknowledgment}

The authors wish to thank Universiti Teknologi PETRONAS for giving the support to publish the work in the journal.

\section{References}

[1] I. Batarseh "Power Electronic Circuits", University of Central Florida, John Wiley \& Sons, Inc., 2004.

[2] A.P. Malvino, "Transistor Circuit Approximation", McGraw-Hill, Inc., $3^{\text {rd }}$ Ed., 2007 (Available: http://www.eng.uwi.tt/depts/elec/staff/rdefour/ee33d/s2_rrchar.html)

[3] F. Mohammed, M.F. Bain, F.H. Ruddell, D. Linton, H.S. Gamble and V.F. Fusco, "A Novel Silicon Schottky Diode for NLTL Applications", IEEE Transactions on Electron Devices, vol. 52, iss. 7, pp. 1384-1391, July. 2005.

[4] B. Ozpineci and L.M. Tolbert, "Characterization of SiC Schottky Diodes at Different Temperatures", IEEE Power Electronics Letters, vol. 1, no. 2, pp. 54-57, Jun. 2003. 
N. Z. Yahaya, et al.

[5] M.S. Chinthavali, B. Ozpineci and L.M. Tolbert, "Temperature-Dependent Characterization of $\mathrm{SiC}$ Power Electronic Devices", IEEE Power Electronic in Transportations, pp. 43-47, Oct. 2004.

[6] M.J. Kearney, M.J. Kelly, A. Condie and L. Dale, "Temperature Dependent Barrier Heights in Bulk Unipolar Diodes Leading to Improved Temperature Stable Performance", IEEE Electronic Letters, vol. 26, iss. 10, pp. 671-672, May 1990.

[7] B.J. Baliga, "Power Semiconductor Device Figure of Merit for High-Frequency Applications", IEEE Electron Device Letters, vol. 10, iss. 10, pp. 455-457, Oct. 1989.

[8] Purdue University Nanoscale Center, Wide Bandgap Semiconductor Devices. (Available: http://www.nanodevices.ecn.purdue.edu/widebandgap.html)

[9] IFM Materials Science Division Linkopins Universitet, "Crystal Structure of Silicon Carbide",(Available:Http://www.ifm.liu.se/matephys/AAnew/research/sicpart/kordina2.ht m), Sep. 2006.

[10] B. Ozpincci and L. M. Tolbert, "Comparison of Wide-Bandgap Semiconductors for Power Electronics Applications", Oak Ridge National Laboratory, Tennessee, Dec. 2003.

[11] A. Ahmed, "Power Electronics for Technology", Purdue University-Calumet, Prentice Hall, 2003.

[12] N.Z. Yahaya and K.K. Chew, "Comparative Study of the Switching Energy Losses between Si PiN and SiC Schottky Diodes", IEEE Proceedings in National Power \& Energy Conference, pp. 216-219, Nov. 2004.

[13] Z. Hui, L.M. Tolbert and B. Ozpineci, "System Modeling and Characterization of SiC Schottky Power Diodes" IEEE Workshops on Computers In Power Electronics, pp. 199204, Jul. 2006.

[14] M. Bhatnagar and B.J. Baiga, "Comparison of $6 \mathrm{H}-\mathrm{SiC}, 3 \mathrm{C}-\mathrm{SiC}$ and $\mathrm{Si}$ for Power Devices", IEEE Transactions on Electronic Devices, vol 40, iss. 3, pp. 645-655, Mar. 1993.

[15] M.S Chintivali, B. Ozpineci and L.M Tolbert, "High-Temperature and High-Frequency Performance Evaluation of 4H-SiC Unipolar Power Devices", IEEE Applied Power Electronics Conference and Exposition, vol. 1, pp. 322-328, Mar. 2005.

[16] L.Scheick, L. Selva and H. Becker, "Displacement Damage-Induced Catastrophic Second Breakdown in Silicon Carbide Schottky Power Diodes", IEEE Transactions on Nuclear Science, vol. 51, iss. 6, pp. 3193- 3200, Dec. 2004.

[17] R.L. Libby, T. Ise and L. Sison, "Switching Characteristics of SiC Schottky Diodes in a Buck DC-DC Converter" Proceedings in. Electronic and Communications Engineering Conference,(Available: http://www.dilnet.upd.edu.ph/ irc/pubs/local/libbyswitching.pdf), Oct. 2006. 